\title{
Influence of Hamstring Tightness in Pelvic, Lumbar and Trunk Range of Motion in Low Back Pain and Asymptomatic Volunteers during Forward Bending
}

\author{
Felipe Jose Jandre Reis ${ }^{1,2}$, Adriana Ribeiro Macedo ${ }^{1}$ \\ ${ }^{1}$ Department of Physiotherapy, Federal Institute of Rio de Janeiro, Rio de Janeiro, Brazil \\ ${ }^{2}$ Department of Clinical Medicine, Federal University of Rio de Janeiro, Rio de Janeiro, Brazil
}

\begin{abstract}
Study Design: Cross-sectional study.
Purpose: To verify the association of hamstring tightness and range of motion in anterior pelvic tilt (PT), lumbar motion (LM), and trunk flexion (TF) during forward bending.

Overview of Literature: Increased hamstring stiffness could be a possible contributing factor to low back injuries. Clinical observations have suggested that hamstring tightness influences lumbar pelvic rhythm. Movement restrictions or postural asymmetry likely lead to compensatory movement patterns of the lumbar spine, and subsequently to increased stress on the spinal soft tissues and an increased risk of low back pain (LBP).

Methods: Hamstring muscle tightness was measured using the self-monitored active knee extension (AKE) test. A bubble inclinometer was used to determine the range of motion of PT, LM, and TF during forward bending. Statistical analysis included descriptive statistics, comparisons between groups and a correlation between hamstring tightness (AKE) and anterior PT, TF, and regional LM with $p \leq 0.05$.

Results: The LBP group was composed of 36 participants, and the asymptomatic group consisted of 32 participants. The mean for PT in the control group was $66.7^{\circ}, 64.5^{\circ}$ for $\mathrm{LM}$ and $104.6^{\circ}$ for TF. Respective values in the symptomatic group were $57.0^{\circ}, 79.8^{\circ}$, and $82.2^{\circ}$.

Conclusions: Participants with LBP showed restriction in the pelvis and TF range of motion, but had higher amplitudes in the lumbar spine during forward bending.
\end{abstract}

Keywords: Low back pain; Muscle tonus; Range of motion; Spine

\section{Introduction}

Low back pain (LBP) is an extremely common musculoskeletal complaint that occurs in upwards of $80 \%$ of persons at some point in their lifetimes [1-3]. This condition is the main cause of years lived with a disability (YLDs)
[3-5]. Definitions and prevalence estimates vary among different populations due to cultural, linguistic, methodological and experiential variabilities [5-6].

Muscle tightness may be linked to postural disturbances. Both can contribute to multiple musculoskeletal conditions $[2,6,7]$. Reduced extensibility resulting from

Received Nov 26, 2014; Revised Dec 16, 2014; Accepted Dec 18, 2014

Corresponding author: Felipe Jose Jandre Reis

Department of Physiotherapy, Federal Institute of Rio de Janeiro, Campus Realengo-Rua Carlos Wenceslau,

343, Realengo CEP 21715-000, Rio de Janeiro, Brasil

Tel: +55-21-3463-4497, Fax: +55-21-3463-4497, E-mail: felipe.reis@ifrj.edu.br 
increased hamstring stiffness could be a possible contributing factor to low back injuries $[7,8]$. Clinical observations have suggested that hamstring tightness influences the lumbar pelvic rhythm and may be associated with modifications in the sagittal spine curvatures during trunk flexion (TF) [8]. Considering that forward bending is one of the most common movements in daily activities, shortened hamstrings may increase the risk of injury to the spine from mechanical stresses [7,9-11].

This study sought to verify the association of hamstring tightness and the range of motion of anterior pelvic tilt (PT), lumbar and TF during forward bending in chronic LBP and asymptomatic participants.

\section{Materials and Methods}

The protocol of this cross-sectional study was previously approved by the local ethics committee, Instituto Federal do Rio de Janeiro (IFRJ) and all patients were given oral and written information before they agreed to participate.

\section{Subjects}

Students from a university population volunteered for this research. Recruitment took place on each day of testing over the course of one week by asking passing students to participate. In the LBP group, the inclusion criterion was the presence of pain at least one day per week in the last 6 months. In the control group, asymptomatic volunteers had no history of back pain in the last 12 months and no restrictions in bending forward. Subjects were excluded from the study if they experienced any significant knee, low back or pelvic pain during the examination, had any history of lumbar or lower limb fracture or surgery, had any lower limb discrepancy $(\geq 2.0 \mathrm{~cm})$, radiculopathy or radicular pain, were currently pregnant, had given birth within the last 12 months or had any balance disorders (positive Romberg test). Subjects with lumbar radiculopathy were also excluded by clinical examination by a physiotherapist including a clinical neurological examination of the lumbar spine and lower limbs.

\section{Procedures}

\section{1) Hamstring muscle tightness}

This was measured using the self-monitored active knee extension (AKE) test described previously $[12,13]$. The subjects lay supine on a bench and flexed their right knee and hip to $90^{\circ}$. They monitored the position of the femur with their right hand and were instructed not to allow the femur to move away from the hand at any point during the test. The participants were instructed to extend their right leg as far as possible, keeping their foot relaxed. The end position was held for 5 second, and the angle of knee extension was measured using a standard plastic goniometer (Physiomed, Manchester, UK). The centre of the goniometer was positioned over the lateral knee joint line and the goniometer arms were positioned along the femur and fibula. The AKE test was used to measure both the right and left knees. The ankle of the tested leg was restrained in plantar flexion to avoid adverse neutral tension. The endpoint for the AKE test was determined by the criterion of pain in the hamstring muscles. Three trials were conducted for each leg and the average of the three trials on each side was used for subsequent analysis. Hamstring tightness was considered if the volunteer was unable to keep the hip flexed at $90^{\circ}$ and the knee totally extended $\left(180^{\circ}\right)$.

\section{2) Bony landmarks}

With the subject in the prone position, the L5 was located by palpating intervening vertebrae from the $\mathrm{S} 2$, which is at the level of the posterior superior iliac spine (PSIS), and by checking to make sure that the iliac crest was aligned at approximately L4-L5. In the same manner, the T12 was found by palpating intervening vertebrae up from the S2 and was checked by palpating intervening vertebrae downward from the T7, which is at the level of the inferior angle of the scapula. The T12-L1 and L5-S1 interspinous spaces were marked on the skin.

\section{3) Range of motion during forward bending}

The inclinometer technique described by Mayer et al. [14] was adopted for measuring the flexion range of motion (ROM) of the lumbar spine. The inclinometer with the two-point contact at its base was used BASELINE Gravity Inclinometer (Fabrication Enterprises Inc., Irvington, NY, USA). It was positioned in the L5-S1 landmark to measure the anterior PT movement. Then, the inclinometer was positioned at T12-L1 to measure the total forward bending or TF. Regional lumbar motion (LM) was acquired by subtracting the resulting TF and PT. The participants were asked first to stand in their usual, relaxed posture. The baseline inclinometer values were recorded 
Table 1. Results of pelvic tilt, lumbar motion, trunk flexion range of motion and anterior knee extension test in chronic low back pain and asymptomatic volunteers

\begin{tabular}{|c|c|c|c|c|c|c|c|}
\hline \multirow{2}{*}{ Variable } & \multicolumn{3}{|c|}{ Chronic low back pain (n=35) } & \multicolumn{3}{|c|}{ Asymptomatic (n=32) } & \multirow{2}{*}{$p$-value } \\
\hline & Mean & SD & $95 \% \mathrm{Cl}$ & Mean & SD & $95 \% \mathrm{Cl}$ & \\
\hline Pelvic tilt ROM & 57.0 & 15.3 & $51.3-62.2$ & 66.7 & 19.4 & $59.7-73.7$ & 0.02 \\
\hline Lumbar ROM & 79.8 & 34.3 & 68.0-91.6 & 64.5 & 34.8 & $52.1-76.9$ & 0.07 \\
\hline Trunk flexion ROM" & 82.2 & 39.7 & $68.3-95.9$ & 104.6 & 29.3 & $93.4-114.6$ & 0.01 \\
\hline \multicolumn{8}{|l|}{ AKE } \\
\hline Right & 154.9 & 14.5 & 149.9-159.9 & 155.5 & 17.0 & $149.3-161.6$ & 0.87 \\
\hline Left & 153.7 & 15.0 & 148.5-158.9 & 154.3 & 19.0 & $147.5-161.2$ & 0.88 \\
\hline
\end{tabular}

$\mathrm{SD}$, standard deviation; $\mathrm{Cl}$, confidence interval; $\mathrm{ROM}$, range of motion; AKE, active knee extension test.

a) Levene test: equal variances not assumed $(p>0.05)$.

Table 2. Results of pelvic tilt, lumbar motion, trunk flexion range of motion and anterior knee extension test according to gender

\begin{tabular}{|c|c|c|c|c|c|c|}
\hline \multirow{2}{*}{ Variable } & \multicolumn{3}{|c|}{ Female $(n=26)$} & \multicolumn{3}{|c|}{ Male $(n=41)$} \\
\hline & Mean \pm SD & Median & $95 \% \mathrm{Cl}$ & Mean $\pm S D$ & Median & $95 \% \mathrm{Cl}$ \\
\hline Pelvic tilt ROM & $64.6 \pm 14.6$ & 65 & $55.9-73.2$ & $59.7 \pm 15.3$ & 60 & $54.9-64.6$ \\
\hline Lumbar flexion ROM & $67.6 \pm 34.8$ & 60 & $53.6-81.7$ & $75.6 \pm 35.1$ & 60 & $64.5-86.7$ \\
\hline Trunk flexion ROM & $97.6 \pm 35.3$ & 107 & $83.4-111.9$ & $89.5 \pm 37.4$ & 100 & 77.6-101.3 \\
\hline \multicolumn{7}{|l|}{$A K E^{a)}$} \\
\hline Right & $164.6 \pm 13.2$ & 160 & 159.3-170.0 & $149.2 \pm 14.1$ & 150 & $144.7-153.6$ \\
\hline Left & $165.7 \pm 13.4$ & 170 & $160.3-171.2$ & $146.6 \pm 14.6$ & 145 & $141.9-151.2$ \\
\hline
\end{tabular}

$\mathrm{SD}$, standard deviation; $\mathrm{Cl}$, confidence interval; $\mathrm{ROM}$, range of motion; AKE, active knee extension test.

${ }^{\text {a) }} p=0.001$ (independent $t$-test).

at T12-L1 and L5-S1. Thereafter, the participants were asked to bend forward to the end of their maximal active range. The readings at $\mathrm{T} 12-\mathrm{L} 1$ and $\mathrm{L} 5-\mathrm{S} 1$ were measured in the maximum flexed position [15].

\section{4) Data analyses}

Data were entered into a database and analyzed using IBM SPSS ver. 20 (IBM Co., Armonk, NY, USA) Descriptive statistics were calculated for demographic information. The Kolmogorov-Smirnov test was used to evaluate if axial alignment followed a normal distribution. A comparison between groups was made using the Student $t$-test. A Pearson correlation ( $r$ ) analysis of the hamstring tightness (AKE) and the anterior PT, TF and regional LM was calculated. The level of significance was set at 0.05 .

\section{Results}

The sample consisted of 67 volunteers selected by conve- nience. The sample was comprised of 41 males (mean age, 27.6 years) and 26 females (mean age, 28.2 years). The average weight and height in males were $78.5 \mathrm{~kg}\left(x_{\min }=63\right.$ $\mathrm{kg}, x_{\max }=96 \mathrm{~kg}$; standard deviation [SD], $8.7 \mathrm{~kg}$ ) and 1.77 $\mathrm{m}\left(x_{\min }=1.67 \mathrm{~m}, x_{\max }=1.92 \mathrm{~m}\right.$; SD, $\left.0.05 \mathrm{~m}\right)$, respectively. The respective values in females were $60.8 \mathrm{~kg}\left(x_{\min }=50 \mathrm{~kg}\right.$, $x_{\text {max }}=75 \mathrm{~kg}$; SD, $\left.6.8 \mathrm{~kg}\right)$ and $1.62 \mathrm{~m}\left(x_{\min }=1.54 \mathrm{~m}, x_{\max }=1.76\right.$ $\mathrm{m}$; SD, $0.05 \mathrm{~m}$ ). The mean AKE for all subjects was $155.2^{\circ}$ (right knee) and $154^{\circ}$ (left knee), $115.5^{\circ}$ for TF, $50.0^{\circ}$ for LM and $65.1^{\circ}$ for PT. Table 1 presents PT, LM, TF, and AKE according to gender.

The asymptomatic volunteer group was composed of 23 males (26.8 years; SD, 6.0 years) and 9 females (31.0 years; SD, 9.2 years). The symptomatic group consisted of 18 males (25.7 years; SD, 5.7 years) and 17 females (26.2 years; SD, 7.6 years). The mean for PT in the control group was $66.7^{\circ}, 64.5^{\circ}$ for $\mathrm{LM}$ and $104.6^{\circ}$ for TF. The respective values for the symptomatic group were $57.0^{\circ}$, $79.8^{\circ}$, and $82.2^{\circ}$ (Table 2 ). 
Table 3. Correlation of pelvic tilt, lumbar motion, trunk flexion range of motion and hamstring tightness in chronic low back pain and asymptomatic volunteers

\begin{tabular}{|c|c|c|c|c|c|c|}
\hline \multirow{2}{*}{ Variable } & \multicolumn{3}{|c|}{ Chronic low back pain $(n=35)$} & \multicolumn{3}{|c|}{ Asymptomatic (n=32) } \\
\hline & Pelvic & Lumbar & Trunk & Pelvic & Lumbar & Trunk \\
\hline \multicolumn{7}{|c|}{ Active knee extension test } \\
\hline Right & $0.41^{a)}$ & -0.04 & $0.44^{\mathrm{bl}}$ & $0.62^{b)}$ & 0.29 & 0.12 \\
\hline Left & $0.44^{\mathrm{bl}}$ & 0.01 & 0.29 & $0.68^{b)}$ & 0.16 & 0.23 \\
\hline
\end{tabular}

The table shows the Pearson correlation coefficients.

${ }^{a)}$ Correlation is significant at the 0.05 level; ${ }^{\text {b) }}$ Correlation is significant at the 0.01 level.

The correlation between hamstring tightness and range of motion is presented in Table 3. Hamstring tightness showed a moderate correlation, with PT movement being higher in the control group. A very weak correlation was observed with LM in the control and the symptomatic group. TF showed a moderate correlation with hamstring tightness in LBP volunteers.

\section{Discussion}

The hamstring muscles are of significant clinical interest in the prevention and treatment of LBP, especially because of the probable indirect involvement of tight hamstrings in the pathogenesis of LBP [16-18]. It is not clear whether or not hamstring extensibility is a predictive factor in the etiology of LBP, or whether it is part of the chronic adaptation to the onset of symptoms, which is possibly associated with other maladaptive postural strategies $[8,16,19$ 21].

In this study, participants in the LBP group showed a lower range of pelvic motion when compared with asymptomatic volunteers, although no statistical difference in hamstring tightness between the groups was observed. This finding has been reported previously by other authors. Halbertsma et al. [17], with 20 participants in a LBP group and 20 in a healthy control group, found that the LBP group showed a significant restriction in the range of motion and in the extensibility of the hamstrings compared with the control group, and no significant difference in hamstring muscle stiffness could be assessed between the groups. Johnson and Thomas [22] reported no significant correlation between hamstring flexibility and hip and lumbar joint excursions during forward bending tasks in LBP or recovered groups. In addition, it is possible that the LBP volunteers performed the forward bending movement in a start position with the pelvis in posterior rotation $[21,23]$. Another possibility to explain restriction in pelvic movement in this group is that greater electrical activity in the hamstring muscles has been shown in LBP subjects $[23,24]$.

Movement restrictions or postural asymmetry is believed to lead to compensatory movement patterns of the lumbar spine, and subsequently to increased stress on the spinal soft tissues and an increased risk of injury [7,23]. In this sample, the LBP participants showed restrictions in pelvic movement, but higher amplitude in lumbar flexion. It has been postulated that, when two body segments move, the segment that is more mobile will move first. This phenomenon has been termed relative flexibility [24]. In the same way, Carregaro and Coury [21], in a study to evaluate the influence of reduced hamstring flexibility on trunk and pelvic movement strategies during manual handling tasks, found that subjects with reduced flexibility presented higher trunk movement amplitudes and a restriction in pelvic movements during handling tasks.

Hamstring tightness showed a moderate correlation with PT movement. This finding can be explained because it is thought that hamstring muscles draw the pelvis into posterior rotation $[7,8,16]$. Our data showed that hamstring extensibility significantly influences pelvic position during maximal TF due to their attachment to the ischial tuberosity on the pelvis. This finding is supported by previous studies [8,9]. However, Norris and Matthews [10] observed no correlation between hamstring length and PT during forward bending in a healthy student population. The main difference from our findings was that the authors studied the anterior PT during forward bending with an amplitude restricted by a bar positioned at the height of individual's tibial length. We believe that this limitation in forward bending should reduce the influence 
of the hamstring in pelvic movement. In this context, a method that could restrict pelvic movement during TF limits the influence of hamstring flexibility $[8,15]$.

With respect to trunk flexion, the LBP participants showed a reduction in range of motion compared to asymptomatic participants. This finding is supported by Gajdosik et al. [8], who reported that the pelvic angle and flexion range of motion, and the thoracic angle and flexion ROM, were influenced by hamstring length during forward bending.

Controversial findings in the literature may be due to different definitions of hamstring tightness or even the method used to assess muscle extensibility $[7,16,17]$. Previous studies used supine hip flexion leg lift angle or straight leg raise (SLR) to measure hamstring tightness $[8,16,22,25-28]$. We preferred to use the AKE test, because it involves less motion in the lumbar spine and pelvis. Compensatory movements in the pelvis and lumbar region occur during SLR, and therefore, it should contribute to increase hip flexion [25]. It is also important to highlight that the test presents excellent intertester and intratester correlation (ICC, 0.99) [13].

To examine the influence of hamstring tightness as a risk factor for LBP over the years [29], the authors assessed flexibility and strength in adolescents and followed them for 25 years to assess their predictive value for LBP. According to the authors, there was no association between hamstring tightness and the incidence of LBP.

It is important to note the methodological limitations of this study. Primarily, in this cross-sectional design, it was not possible to establish a cause-effect relation between hamstring tightness and LBP. Once it was shown that there was no difference in hamstring tightness between the groups, it was possible that LBP participants presented pelvic positional alterations. We did not assess their standing posture to verify this, or the influence of the hamstring on the pelvis and lumbar region, before the forward bending movement. The influence of other muscles, such as the hip or back extensors, was not evaluated. In addition, during the forward bending motion of the pelvis, the lumbar and trunk could have been more accurately assessed using video analysis. Consequently, further research into any association is necessary.

Accepting these limitations, the correlation between pelvic movement and hamstring tightness is an important finding in clinical practice. This data suggests that muscle intervention, such as hamstring stretching, may be useful to restore pelvic movement. LBP patients showed higher restriction in $\mathrm{PT}$ and greater mobility in lumbar region during forward bending. This finding could be explained by the compensatory increase in the range of motion in the lumbar region during the forward bending movement. In clinical practice, limitation in PT and greater range of motion in lumbar region should be an indicative of low back stabilization exercises and pelvic mobilization. Further studies that investigate the hamstring tightness as a risk factor to LBP are needed.

\section{Conclusions}

Hamstring tightness had no influence pelvic motion in both groups during forward bending. No difference was observed between LBP and asymptomatic volunteers in hamstring tightness. Concerning range of motion, the PT and TF were higher in the asymptomatic group and LM in LBP participants.

\section{Conflict of Interest}

No potential conflict of interest relevant to this article was reported.

\section{References}

1. Sandler RD, Sui X, Church TS, Fritz SL, Beattie PF, Blair SN. Are flexibility and muscle-strengthening activities associated with a higher risk of developing low back pain? J Sci Med Sport 2014;17:361-5.

2. Dionne CE, Dunn KM, Croft PR, et al. A consensus approach toward the standardization of back pain definitions for use in prevalence studies. Spine (Phila Pa 1976) 2008;33:95-103.

3. Deyo RA, Dworkin SF, Amtmann D, et al. Report of the NIH Task Force on research standards for chronic low back pain. Pain Med 2014;15:1249-67.

4. Murray CJ, Atkinson C, Bhalla K, et al. The state of US health, 1990-2010: burden of diseases, injuries, and risk factors. JAMA 2013;310:591-608.

5. Hoy D, Bain C, Williams G, et al. A systematic review of the global prevalence of low back pain. Arthritis Rheum 2012;64:2028-37.

6. Sahrmann S. Diagnosis and treatment of movement impairment syndromes. St. Louis (MO): Mosby; 2002. 
7. Esola MA, McClure PW, Fitzgerald GK, Siegler S. Analysis of lumbar spine and hip motion during forward bending in subjects with and without a history of low back pain. Spine (Phila Pa 1976) 1996;21:71-8.

8. Gajdosik RL, Hatcher CK, Whitsell S. Influence of short hamstring muscles on the pelvis and lumbar spine in standing and during the toe-touch test. Clin Biomech (Bristol, Avon) 1992;7:38-42.

9. Lopez-Minarro PA, Alacid F. Influence of hamstring muscle extensibility on spinal curvatures in young athletes. Sci Sports 2010;25:188-93.

10. Norris CM, Matthews M. Correlation between hamstring muscle length and pelvic tilt range during forward bending in healthy individuals: an initial evaluation. J Bodyw Mov Ther 2006;10:122-6.

11. Nachemson AL. Disc pressure measurements. Spine (Phila Pa 1976) 1981;6:93-7.

12. Norris CM, Matthews M. Inter-tester reliability of a self-monitored active knee extension test. J Bodyw Mov Ther 2005;9:256-9.

13. Gajdosik R, Lusin G. Hamstring muscle tightness: reliability of an active-knee-extension test. Phys Ther 1983;63:1085-8.

14. Mayer TG, Tencer AF, Kristoferson S, Mooney V. Use of noninvasive techniques for quantification of spinal range-of-motion in normal subjects and chronic low-back dysfunction patients. Spine (Phila Pa 1976) 1984:9:588-95.

15. Ng JK, Kippers V, Richardson CA, Parnianpour M. Range of motion and lordosis of the lumbar spine: reliability of measurement and normative values. Spine (Phila Pa 1976) 2001;26:53-60.

16. Raftry SM, Marshall PW. Does a 'tight' hamstring predict low back pain reporting during prolonged standing? J Electromyogr Kinesiol 2012;22:407-11.

17. Halbertsma JP, Goeken LN, Hof AL, Groothoff JW, Eisma WH. Extensibility and stiffness of the hamstrings in patients with nonspecific low back pain. Arch Phys Med Rehabil 2001;82:232-8.

18. Marshall PW, Mannion J, Murphy BA. Extensibility of the hamstrings is best explained by mechanical components of muscle contraction, not behavioral measures in individuals with chronic low back pain. PM R 2009;1:709-18.

19. Li Y, McClure PW, Pratt N. The effect of hamstring muscle stretching on standing posture and on lumbar and hip motions during forward bending. Phys Ther 1996;76:836-45.

20. Fox M. Effect on hamstring flexibility of hamstring stretching compared to hamstring stretching and sacroiliac joint manipulation. Clin Chiropr 2006;9:2132.

21. Carregaro RL, Coury HJ. Does reduced hamstring flexibility affect trunk and pelvic movement strategies during manual handling? Int J Ind Ergon 2009; 39:115-20.

22. Johnson EN, Thomas JS. Effect of hamstring flexibility on hip and lumbar spine joint excursions during forward-reaching tasks in participants with and without low back pain. Arch Phys Med Rehabil 2010;91:1140-2.

23. Van Dillen LR, Gombatto SP, Collins DR, Engsberg JR, Sahrmann SA. Symmetry of timing of hip and lumbopelvic rotation motion in 2 different subgroups of people with low back pain. Arch Phys Med Rehabil 2007;88:351-60.

24. Rose SJ, Sahrmann SA, Norton BT. Quantitative assessment of lumbar-pelvic rhythm. Phys Ther 1988; 68:824.

25. Mooney V, Robertson J. The facet syndrome. Clin Orthop Relat Res 1976;(115):149-56.

26. Congdon R, Bohannon R, Tiberio D. Intrinsic and imposed hamstring length influence posterior pelvic rotation during hip flexion. Clin Biomech (Bristol, Avon) 2005;20:947-51.

27. Massoud Arab A, Reza Nourbakhsh M, Mohammadifar A. The relationship between hamstring length and gluteal muscle strength in individuals with sacroiliac joint dysfunction. J Man Manip Ther 2011;19:510.

28. Stutchfield BM, Coleman S. The relationships between hamstring flexibility, lumbar flexion, and low back pain in rowers. Eur J Sport Sci 2006;6:255-60.

29. Mikkelsson LO, Nupponen H, Kaprio J, Kautiainen H, Mikkelsson M, Kujala UM. Adolescent flexibility, endurance strength, and physical activity as predictors of adult tension neck, low back pain, and knee injury: a 25 year follow up study. Br J Sports Med 2006;40: 107-13. 